\title{
Tumores gastrointestinales estromales (G IST): Experiencia del Servicio de Cirugía del Hospital Clínico de la U niversidad de Chile entre 1999 y 2005
}

\author{
Italo Braghetto ${ }^{1}$, Francisco J Parada ${ }^{1}$, G onzalo Cardemil ${ }^{1}$, \\ Attila Csendes ${ }^{1}$, Eduardo Fernández ${ }^{1}, 0$ wen Korn ${ }^{1}$, \\ Marcos Ramírez ${ }^{1}$, Laura Carreño ${ }^{2}$, G ladys Smok², \\ Juan Carlos Molina a, Hanns Lembacha. \\ Gastrointestinal stromal tumors. \\ Review of 15 patients
}

Background: Gastrointestinal stromal tumors (GIST) are the most common mesenchymatous tumors of the digestive tract. The pathological diagnosis is based on microscopy and immunohistochemistry. Aim: To review the experience of our surgical unit in patients with GIST. Material and methods: Review of medical records of 15 patients (aged $66 \pm 13$ years, 11 women), with a pathological diagnosis of GIST, treated between 1999 and 2005. Results: The main presenting symptoms were melena in $40 \%$, hematemesis in $20 \%$, abdominal pain in $60 \%$ and anemia in $13 \%$. In only one patient, the tumor appeared as an incidentaloma. All patients underwent upper gastrointestinal endoscopy. A CAT scan was done in $87 \%$, a barium swallow in $60 \%$ and a digestive endosonography in $20 \%$. Thirteen tumors were located in the stomach and two in the small bowel. Mean tumor diameter was $5.3 \pm 1.7 \mathrm{~cm}$. Surgical management was a tumor resection in $40 \%$, a partial gastrectomy in $27 \%$, a total gastrectomy in $20 \%$ and an intestinal excision in the rest. Mean hospital stay was $6.9 \pm 4.2$ days. No postoperative complications were recorded. Conclusions: The main clinical presentation of GIST in this retrospective series was an upper gastrointestinal bleeding. Surgical treatment was devoid of complications (Rev Méd Chile 2007; 135: 551-7).

(Key words: Gastrointestinal hemorrhage; Gastrointestinal stromal tumors; Gastrectomy)

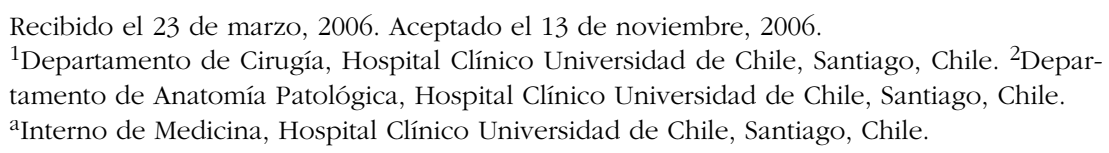

$\mathrm{L}$ os tumores estromales gastrointestinales (GIST), corresponden a una entidad anatomopatológica recientemente descrita como el conjunto de tumores del tracto digestivo provenientes

Correspondencia a: Dr. Italo Braghetto M. Santos Dumont 999, Santiago Chile. Fax: 56-2-9788335. E mail: ibraghet@redclinicauchile.cl del estroma, que expresan en la superficie de sus células receptores tirosin-kinasa con una mutación autoactivante $(\mathrm{c}-\mathrm{kit}+)^{1-5}$.

Este tema, aún en discusión dentro de la especialidad quirúrgica y oncológica, corresponde a una entidad que recién en 1998 comienza a dilucidarse, al establecer el criterio patológico de diagnóstico (c-kit + a inmunohistoquímica) ${ }^{2,5}$. Desde entonces se ha logrado progresivamente 
mayor claridad en temas relacionados al diagnóstico, manejo y pronóstico de estos tumores. Sin embargo, múltiples estudios clínicos principalmente relacionados al tratamiento con anticuerpos monoclonales anti-tirosin-kinasa (IMATINIB ${ }^{\circledR}$ ) y estudios de seguimiento respecto al pronóstico se encuentran aún en curso y se esperan sus resultados para decidir qué conductas diagnósticas y terapéuticas son las más beneficiosas para estos pacientes $^{1}$. Su presentación clínica varía desde asintomático a situaciones de urgencia como hemorragia digestiva de diferente cuantía y sangrados extraluminales con hemoperitoneo.

La serie que presentamos tiene por objetivo describir las conductas de diagnóstico y manejo que se han seguido en el Servicio de Cirugía del Hospital Clínico de la Universidad de Chile desde 1999 a 2006, y compararlas con la última evidencia respecto al tema.

\section{Material y MÉTODO}

Se realizó una revisión retrospectiva de las fichas clínicas de todos los casos registrados como tumor GIST en el Hospital Clínico de la Universidad de Chile hasta el año 2006. Se revisaron los registros clínicos, exámenes e informes de biopsias realizadas por el Servicio de Anatomía Patológica del Hospital. Se incluyeron los pacientes, independientemente de su edad, sexo, o patologías concomitantes, que tuviesen una lesión tumoral compatible y cuya biopsia concluyera tumor GIST como diagnóstico luego de un análisis microscópico, histológico e inmunohistoquímico. Para este último, se consideró como criterio único de inclusión en el estudio, la presencia de un marcador c-kit + , además del registro de CD34 y actina. Posteriormente se revisó el manejo quirúrgico y la evolución de estos pacientes, y se excluyeron quienes no habían sido diagnosticados y tratados en el hospital.

\section{Resultados}

La serie quedó conformada por 15 pacientes, con una mediana de edad de $66 \pm 13$ años. Al analizar por sexo, la muestra está compuesta por 4 hombres, con una media de edad de 65,3 años, y 11 mujeres con una mediana de edad de 59,5 años.
El tiempo promedio de sintomatología fue de 19,9 días previo a la consulta, que en general se realizó en el Servicio de Urgencias del Hospital Clínico de la Universidad de Chile. Sólo uno de los pacientes consultó en policlínico con una tomografía de control y se encontró el tumor como hallazgo. El cuadro predominante de presentación en esta serie fue la hemorragia digestiva alta (melena en $40 \%$ y hematemesis en 20\% de los casos), y el dolor abdominal, de distintas características, presentado por $60 \%$ de los pacientes. Dos de los pacientes (13,3\%) presentaron una anemia crónica producto de una hemorragia oculta del tumor y, como se comentó antes, sólo uno de los pacientes $(6,6 \%)$ se presentó como hallazgo al estudio o control de otra patología.

Dentro de los exámenes de investigación, 100\% de la serie se estudió con endoscopia digestiva alta y biopsia endoscópica. El segundo examen en frecuencia fue la tomografía axial computarizada de abdomen y pelvis, que fue solicitada en $86,6 \%$ de los pacientes. Otro examen frecuente para el estudio fue la radiografía de esófago-estómagoduodeno, practicada en $60 \%$ de los pacientes. Por último, 3 pacientes (20\%) complementaron su estudio con endosonografía digestiva.

La totalidad de los pacientes de la serie tuvo estudio histopatológico e inmunohistoquímico de las lesiones. Este estudio incluyó marcadores como c-kit, CD34, actina, s100, vimentina y desmina, y se consideró la presencia del marcador c-kit + como criterio indispensable para el diagnóstico y la inclusión en el estudio. Además, se registraron CD34 y actina como marcadores complementarios para el diagnóstico. De esta manera, 100\% de la serie resultó positiva para c-kit, 40\% para CD34 y $20 \%$ para actina + (Tabla 1). La Figura 4 muestra un estudio inmunohistoquímico positivo para el marcador c-kit, realizado al tejido biopsiado a uno de los pacientes de la serie.

Con respecto al manejo quirúrgico, en el caso de los tumores de la pared gástrica el procedimiento más realizado fue la tumorectomía, en $40 \%$ de los pacientes. La gastrectomía parcial o segmentaria ocupó el segundo lugar con 26,6\% de los procedimientos, seguida de la gastrectomía total en tercer lugar con $25 \%$. Por otro lado, en los tumores de la pared intestinal, los dos casos presentados en esta serie fueron tratados mediante resección del segmento comprometido con bordes quirúrgicos libres, y posterior anastomosis 
término-terminal. El promedio de días de hospita-

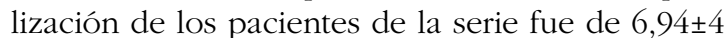
días, sin presentarse complicaciones posoperatorias en ninguno de los pacientes (Tabla 2).
Características del tumor. Trece de los 15 pacientes presentaron ubicación gástrica $(86,6 \%)$ y dos pacientes presentaron tumor en el intestino delgado $(13,3 \%)$, uno de ubicación duodenal (segunda por-

Tabla 1. Síntoma de presentación y estudio de 15 casos de tumor GIST*

\begin{tabular}{|c|c|c|c|c|c|c|c|c|}
\hline Paciente & Sexo & $\begin{array}{l}\text { Edad } \\
\text { (años) }\end{array}$ & Síntoma de Presentación & $\begin{array}{c}\text { Lapso (días) } \\
\text { síntomas cirugía }\end{array}$ & Estudio & \multicolumn{3}{|c|}{ Inmunohistoquímica } \\
\hline 1 & $\mathrm{~F}$ & 50 & Hematemesis & 9 & $\mathrm{EDA}+\mathrm{TAC}+\mathrm{RxEED}+\mathrm{ESD}$ & + & - & + \\
\hline 2 & $\mathrm{~F}$ & 77 & Melena + Sd. Anémico & 25 & $\mathrm{EDA}+\mathrm{TAC}+\mathrm{RxEED}+\mathrm{ESD}$ & + & + & - \\
\hline 3 & $\mathrm{~F}$ & 62 & Hallazgo & 4 & $\mathrm{EDA}+\mathrm{TAC}+\mathrm{RxEED}$ & + & + & + \\
\hline 4 & M & 54 & Melena + Sd. anémico & 10 & $\mathrm{EDA}+\mathrm{TAC}$ & + & + & + \\
\hline 5 & M & 85 & Dolor abdominal & 60 & $\mathrm{EDA}+\mathrm{TAC}+\mathrm{RxEED}$ & + & - & - \\
\hline 6 & $\mathrm{~F}$ & 62 & Dolor abdominal & 139 & $\mathrm{EDA}+\mathrm{TAC}$ & + & + & - \\
\hline 7 & $\mathrm{~F}$ & 66 & Melena + Dolor abdominal & 90 & $\mathrm{EDA}+\mathrm{TAC}+\mathrm{RxEED}$ & + & + & - \\
\hline 8 & $\mathrm{~F}$ & 74 & Hematemesis + melena & 3 & $\mathrm{EDA}+\mathrm{TAC}$ & + & - & + \\
\hline 9 & M & 66 & Melena + Sd. anémico & 180 & EDA & + & - & - \\
\hline 10 & $\mathrm{~F}$ & 73 & Hematemesis + melena & 30 & $\mathrm{EDA}+\mathrm{TAC}$ & + & - & - \\
\hline 11 & M & 45 & Dolor abdominal & 120 & $\mathrm{EDA}+\mathrm{TAC}$ & + & - & - \\
\hline 12 & $\mathrm{~F}$ & 46 & Dolor abdominal & 30 & $\mathrm{EDA}+\mathrm{RxEED}$ & + & - & - \\
\hline 13 & $\mathrm{~F}$ & 45 & Dolor abdominal & 30 & $\mathrm{EDA}+\mathrm{TAC}+\mathrm{RxEED}$ & + & + & - \\
\hline 14 & $\mathrm{~F}$ & 37 & Dolor abdominal & 6 & $\mathrm{EDA}+\mathrm{TAC}+\mathrm{RxEED}$ & + & - & - \\
\hline 15 & $\mathrm{~F}$ & 63 & Dolor abdominal & 3 & $\mathrm{EDA}+\mathrm{TAC}+\mathrm{RxEED}+\mathrm{ESD}$ & + & - & - \\
\hline Promedio & & 62,5 & & 19,9 & & & & \\
\hline DE & & 14,24 & & 52,89 & & & & \\
\hline
\end{tabular}

*EDA: Endoscopia digestiva alta; TAC: Tomografía Axial Computarizada; RxEED: Radiografía Esófago-Estómago-Duodeno; ESD: Endosonografía Diagnóstica; DE: Desviación Estándar.

Tabla 2. M anejo quirúrgico de 15 casos de tumor GIST

\begin{tabular}{|cllcrc|}
\hline Paciente & Manejo quirúrgico & Ubicación GIST & $\begin{array}{c}\text { Diámetro } \\
\text { mayor }\end{array}$ & $\begin{array}{r}\text { Días } \\
\text { Hospitalización }\end{array}$ & $\begin{array}{c}\text { Estado } \\
\text { Actual }\end{array}$ \\
\hline 1 & Tumorectomía gástrica & Pared posterior del cuerpo & 3 & 14 & vivo \\
2 & Gastrectomía segmentaria & Cara anterior del cuerpo & 5 & 6 & vivo \\
3 & Resección intestinal & Yeyuno & 4,5 & 9 & vivo \\
4 & Gastrectomía total & Subcardial & 10 & 16 & vivo \\
5 & Gastrectomía total & Cara anterior & 0,8 & 10 & vivo \\
6 & Gastrectomía parcial & Pared posterior & 8 & 5 & vivo \\
7 & Gastrectomía total & Cuerpo, curvatura menor & 5,5 & 17 & vivo \\
8 & Tumorectomía gástrica & Fondo gástrico & 5,5 & 6 & vivo \\
9 & Gastrectomía subtotal & Subcardial & 6 & 5 & vivo \\
10 & Resección intestinal & Segunda porción del duodeno & 6 & vivo \\
11 & Tumorectomía gástrica & Antro & 5,4 & 9 & vivo \\
12 & Gastrectomía subtotal & Antro & 4,5 & 7 & vivo \\
13 & Tumerctomía Gástrica & Curvatura menor & 6 & 4 & vivo \\
14 & Resección transgástrica laparoscópica & Cara Posterior & 4 & 2 & vivo \\
15 & Resección endoscópica & Cara Posterior del fondo & 5,3 & 6,94 & vivo \\
Promedio & & & 1,76 & 4,25 & \\
DE* & & & & & \\
\hline
\end{tabular}

*DE: Desviación Estándar. 
ción), y el otro de ubicación yeyunal. La ubicación gástrica más frecuente fue el cuerpo gástrico, presente en 7 pacientes $(46,6 \%)$, seguido de la subcardial en 2 pacientes y de la ubicación antral en 2 pacientes. Respecto al diámetro mayor, el tamaño promedio en esta serie fue de $5,3 \pm 1 \mathrm{~cm}$ (Tabla 2).

En la Figura 1 se muestra la visión endoscópica del GIST subcardial el cual fue resecado por vía transgástrica laparoscópica. En la Figura 2 se muestra un gran tumor dependiente de la curvatura mayor gástrica que sangró hacia el peritoneo, procediéndose la extirpación del tumor con resección parcial de la pared gástrica. En la Figura 3 se muestra un GIST del fondo gástrico comprometiendo el ángulo de His. Se procedió a la resección del tumor y gastrorrafia con hemifundoplicatura lateral. El seguimiento posoperatorio de la serie muestra que, a la fecha, la totalidad de los pacientes están asintomáticos sin recidiva de la lesión.

\section{DisCUSIÓN}

Los tumores gastrointestinales estromales (GIST) corresponden a las neoplasias de origen mesenquimático más frecuentes del tracto digestivo ${ }^{7}$. Originalmente fueron descritos y clasificados como un subgrupo de tumores derivados de células musculares ${ }^{5,10,11}$, pero con el advenimiento de técnicas

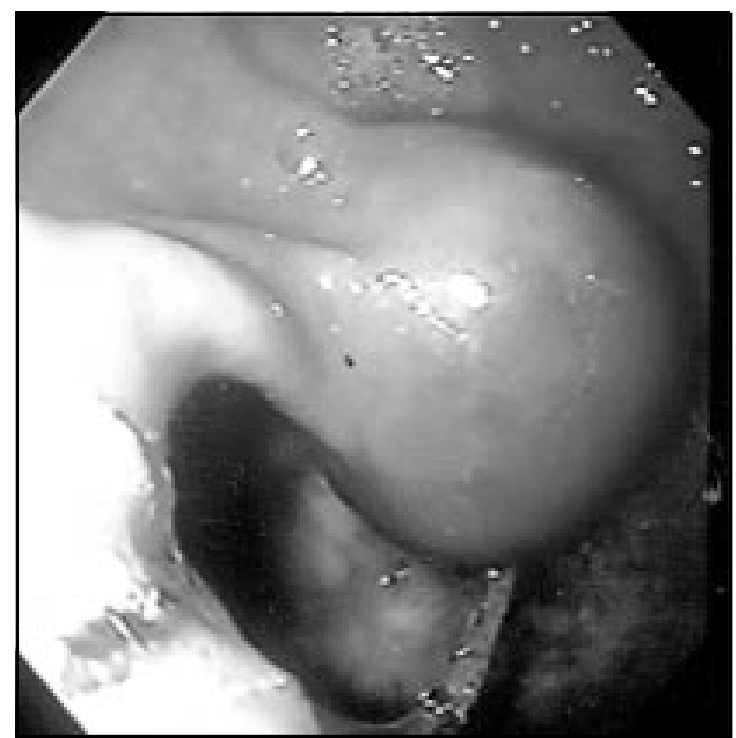

Figura 1. Visión endoscópica de GIST subcardial de pared posterior, sometido a resección transgástrica por vía laparoscópica.

de inmunohistoquímica y microscopia electrónica se observó que éstos poseen menos características de diferenciación muscular que otros leiomiomas originados en otras partes del organismo. Además, se evidenció que algunos de estos tumores presentan marcadores de diferenciación neural (s-100),

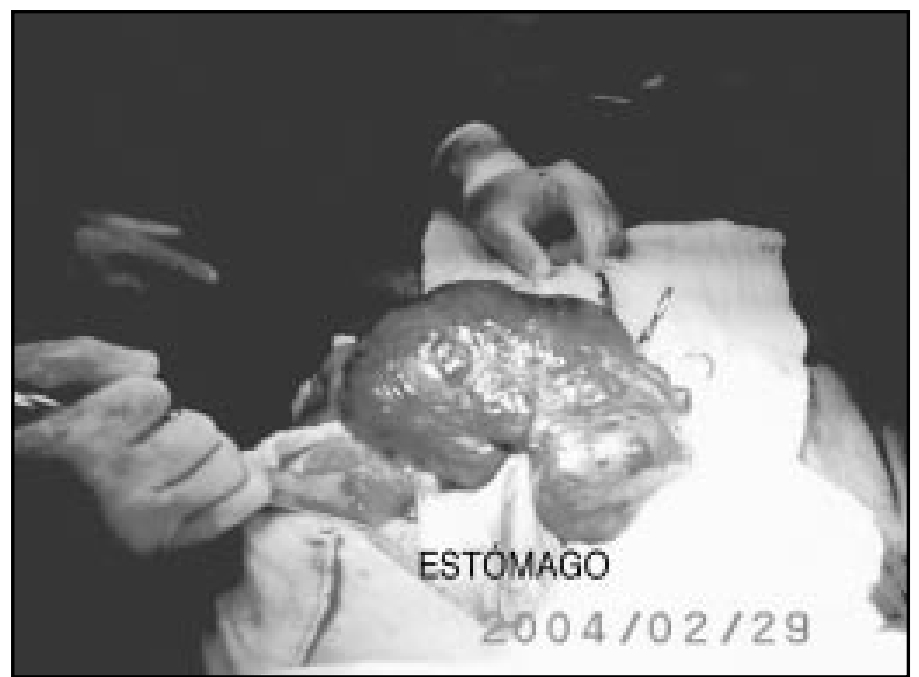

Figura 2. Tumor GIST antral con sangrado intraperitoneal sometido a resección tumoral y resección parcial de pared gástrica. 


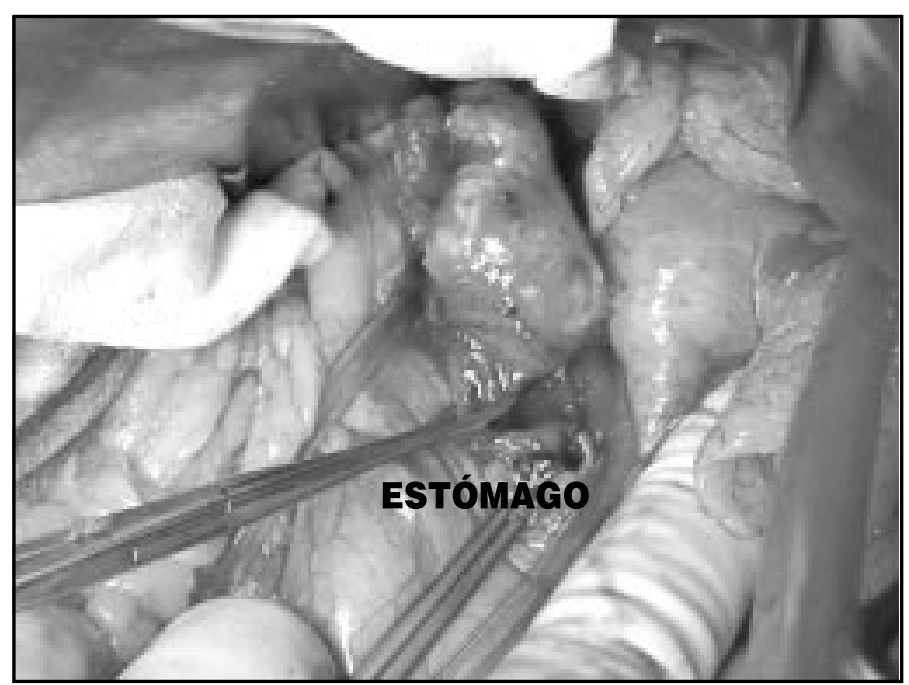

Figura 3. GIST subcardial con dolor y hematemesis sometido a resección del tumor y gastrorrafia.

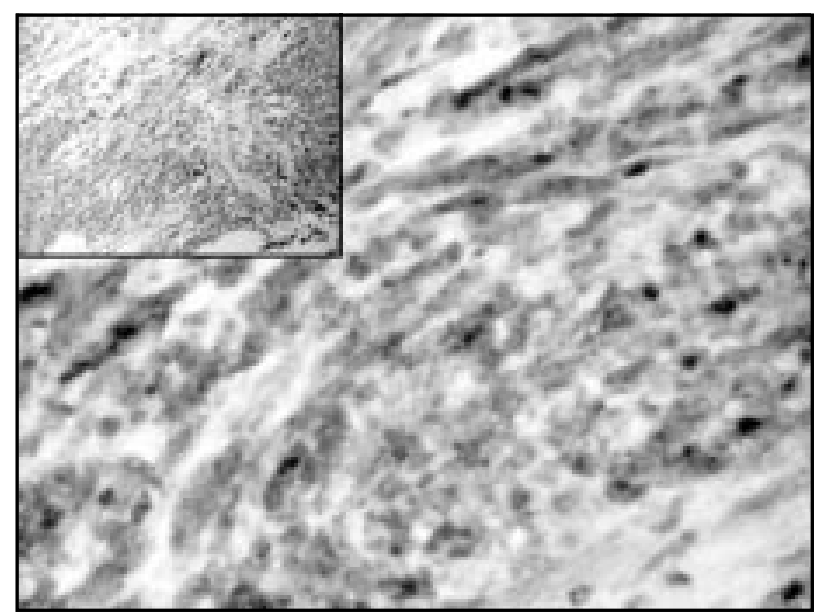

Figura 4. Inmunohistoquímica C-KIT positiva.

por lo que a principios de la década 1980-89, se les describió como una entidad aparte?. Posteriormente se describieron otras características inmunohistoquímicas propias de estas neoplasias, como la expresión de CD 34 (que presentan alrededor de $70 \%$ de los tumores diagnosticados) $)^{3,12-14}$ y finalmente, en 1998, la expresión de receptores de kit, presentes en cerca de $95 \%$ de los pacientes ${ }^{15}$. Este último hallazgo fue el que finalmente quedó determinado como el marcador más importante de tumores GIST. Actualmente se sabe que además de la reactividad para kit, los tumores GIST presentan reactividad para otros marcadores, incluyendo el BCL-2 (80\%), el CD 34 (70\%), actina músculo específica (50\%), alfa actina (35\%), S-100 (10\%) y desmina $(5 \%)^{3,16-18}$. Se ha establecido que el diagnóstico de GIST debe ser hecho por un patólogo experto en sarcomas, y debe ser sustentado por análisis histológico e inmunohistoquímico ${ }^{1}$. Macroscópicamente suelen ser redondeados, uni o 
multilobulados, de bordes netos, bien definidos, color blanquecino, parcial o predominantemente extramurales, centrados en la submucosa, la muscular propia o ambas. Al corte la superficie es lobulada, a veces granular, con zonas de hemorragia y necrosis, raramente presenta zonas de involución quística. A la histología se pueden observar tres patrones distintos: el de células fusiformes (70\%), el epiteloideo (20\%) y el mixto (10\%). El de células fusiformes presenta células con citoplasma levemente eosinofílico, con núcleos ovalados y de apariencia uniforme, dispuestas en forma arremolinada o en fascículos largos. El patrón epiteloideo se compone de células redondas o alargadas, con citoplasma claro levemente eosinofílico, núcleos uniformes redondos u ovalados ${ }^{3}$. El análisis inmunohistoquímico debe incluir CD 117 (c-kit), CD 34, alfa actina, S-100 y desmina.

Epidemiológicamente, corresponde a una entidad poco frecuente, con tasas de 20 por cada millón de habitantes/año; es discretamente más frecuente en hombres (55\% de los pacientes) y el peak de presentación es alrededor de 60 años de edad, pudiendo presentarse en pacientes desde los 8 hasta los 95 años $^{3,4}$. Miettinen et al, en su serie de 1.765 casos, encontraron un promedio de edad de 63 años, con $90 \%$ de sus pacientes sobre 40 años de $e^{e d a d}{ }^{18}$. La distribución por sexo, que en general en otras publicaciones es similar en hombres y mujeres, en nuestra serie es de $73,3 \%$ para mujeres y $26,7 \%$ para hombres. Esta clase de tumores puede presentarse a lo largo de todo el tracto gastrointestinal, ubicándose más frecuentemente en el estómago (60\%), seguido del intestino delgado (25-30\%), recto (5\%), esófago (2\%) y otras localizaciones como el apéndice, páncreas, omento, etc (5\%). Al momento del diagnóstico, generalmente miden entre 2 y 30 $\mathrm{cm}^{3,4}$. La presentación clínica más frecuente es la relacionada con el sangrado digestivo $(54,4 \%)$, manifestándose como anemia, melena o hematemesis, cuya frecuencia relativa varía en las distintas series. Otras formas de presentación incluyen dolor abdominal $(16,8 \%)$ y abdomen agudo $(1,7 \%$, por ruptura del tumor a la cavidad peritoneal) ${ }^{5,7,9}$. Es importante notar que cerca de 18\% de los diagnósticos de GIST fueron incidentales, ya sea por estudio radiológico por otra patología o como hallazgo secundario de una biopsia en una resección quirúrgica por otra causa ${ }^{9}$. Este cuadro clínico fue similar al encontrado en nuestros pacientes.
Dado el patrón de crecimiento predominantemente exofítico de estos tumores, el estudio imagenológico practicado se basó principalmente en endoscopia digestiva alta (EDA), que se practicó en 100\% de los pacientes y observó lesiones solevantadas de la mucosa digestiva de distinta magnitud. En estas condiciones, la EDA fue también de utilidad para el diagnóstico a través de la biopsia endoscópica de la lesión. En el contexto del diagnóstico endoscópico, la endosonografía prestó utilidad en el estudio de 3 de los pacientes de la serie, al permitir la observación de un compromiso exclusivamente intramural en tumores localizados. La radiografía de esófago-estómago-duodeno se utilizó como estudio inicial, pues demostró lesiones solevantadas de la mucosa de aspecto tumoral que motivaron el estudio ulterior. La tomografía axial computarizada fue realizada en 13 de los 15 pacientes de la serie, y fue fundamental para la caracterización del estadio preoperatorio de la lesión, búsqueda de metástasis, y para la determinación del plan quirúrgico a seguir.

El tratamiento actual para los tumores GIST localizados y resecables es la cirugía. Existen grupos que no recomiendan realizar biopsia con aguja preoperatoria (endoscópica con ultrasonido ni percutánea), debido a las características de friabilidad y probable diseminación. Como estos tumores tienen un patrón exofítico de crecimiento y no tienden a infiltrar, una resección en cuña de la lesión en el estómago o una resección segmentaria del intestino son consideradas como el tratamiento de elección. En los casos en que el tumor primario comprometa mesenterio u omento, el procedimiento debe ser la resección en bloque ${ }^{1,3}$. En el caso de tumores GIST irresecables o metastáticos, se ha planteado con resultados alentadores la terapia con imatinib, un bloqueador del receptor KIT. Se ha demostrado que inhibe su autoactivación, induciendo reducción significativa del tamaño tumoral y control de la enfermedad metastásica. Actualmente su uso está avalado en el caso de tumores irresecables o metastáticos, pero estudios clínicos actualmente en curso buscan validar el uso de imatinib como terapia adyuvante o neoadyuvante. Estas nuevas estrategias terapéuticas tendrían como objetivo ampliar el grupo de pacientes candidatos a un tratamiento quirúrgico definitivo y mejorar el pronóstico posquirúrgico, disminuyendo la incidencia de recidivas y diseminación ${ }^{19}$.

El pronóstico de los tumores GIST es variable. Existen variedades benignas y malignas del tumor, y 
su comportamiento va desde pequeños nódulos mitóticamente inactivos hasta tumores metastásicos con amplia diseminación abdominal. La experiencia acumulada hasta ahora en estudios de seguimiento indica que cada tumor en particular tiene su índice de malignidad de acuerdo a su ubicación (GIST gástricos

\section{REFERENCIAS}

1. Blay Jy, Bonvalot S, Casali P, Choi H, DebiecRichter M, Dei Tos AP et al. Consensus meeting for the management of gastrointestinal stromal tumors. Report of the GIST Consensus Conference of 20-21 March 2004, under the auspices of ESMO. Ann Oncol 2005; 16: 566-78.

2. Dematteo RP, Heinrich MC, El-Rifai WM, Demetri G. Clinical management of gastrointestinal stromal tumors: before and alter STI-571. Hum Pathol 2002; 33: 466-77.

3. Fletcher CD, Berman JJ, Corless C, Gorstein F, Lasota J, Longley BJ et al. Diagnosis of gastrointestinal stromal tumors: A consensus approach. Hum Pathol 2002; 33: 459-65.

4. Miettinen M, El-Rifai W, Sobin L, Lasota J. Evaluation of malignancy and prognosis of gastrointestinal stromal tumors: a review. Hum Pathol 2002; 33: $478-83$.

5. Heinrich MC, Corless CL. Gastric GI stromal tumors (GISTs): the role of surgery in the era of targeted therapy. J Surg Oncol 2005; 90: 195-207.

6. Prakash S, Sarran L, Socci N, DeMatteo RP, Eisenstat J, Greco AM et al. Gastrointestinal stromal tumors in children and young adults: a clinicopathologic, molecular, and genomic study of 15 cases and review of the literature. J Pediatr Hematol Oncol 2005; 27: 179-87.

7. Judson I. Gastrointestinal stromal tumours (GIST): biology and treatment. Ann Oncol 2002; 13 Suppl 4: 287-9.

8. Neuhaus SJ, Clark Ma, Hayes AJ, Thomas JM, Judson I. Surgery for gastrointestinal stromal tumour in the post-imatinib era. ANZ J Surg 2005; 75: 165-72.

9. Bucher P, Villiger P, Egger JF, Buhler LH, Morel P. Management of gastrointestinal stromal tumors: from diagnosis to treatment. Swiss Med Wkly 2004; 134: 145-53. tienden a ser más benignos), índice mitótico (el límite se ha puesto en 5 mitosis en 50 campos de mayor aumento), y tamaño del tumor (en general se considera $5 \mathrm{~cm}$ como límite, en intestino delgado este límite se reduce a $2 \mathrm{~cm}$ ), y que de acuerdo a esto se debe plantear un seguimiento posoperatorio adecuado ${ }^{4}$.

10. Appelman HD. Smooth muscle tumors of the gastrointestinal tract. What we know now that Stout didn't know. Am J Surg Pathol 1986; 10: 8399.

11. Appelman HD, Helwig EB. Sarcomas of the stomach. Am J Clin Pathol 1977; 67: 2-10.

12. Mikhael AI, Bacchi CE, Zarbo RJ. CD34 expression in stromal tumors of the gastrointestinal tract. Appl Immunohistochem 1994; 2: 89-93.

13. Miettinen M, Virolainen M, Sarlomo-Rikala M. Gastrointestinal stromal tumors: Value of CD34 antigen in their identification and separation from true leiomyomas and schwannomas. Am J Surg Pathol 1995; 19: 207-16.

14. Miettinen M, Majidi M, Lasota J. Pathology and diagnostic criteria of gastrointestinal stromal tumours (GISTs): a review. Eur J Cancer 2002; 38: 39-51.

15. Hirota S, Isozaki K, Moriyama Y, Hashimoto K, Nishida T, Ishiguro S ET AL. Gain-of-function mutations ok c-kit in human gastrointestinal stromal tumors. Science 1998; 23: 279: 577-80.

16. Miettinen M, Lasota J. Gastrointestinal stromal tumors-Definition, clinical, histological, immunohistochemical, and molecular genetic features and differential diagnosis. Virchows Archiv 2001; 438: 1-12.

17. Corless CL, Fletcher JA, Heinrich MC. Biology of gastrointestinal stromal tumors. J Clin Oncol 2004; 22: 3813-25.

18. Miettinen M, Sobin LH, Lasota J. Gastrointestinal stromal tumors of the stomach: A clinicopathologic, immunohistochemical, and molecular genetic study of 1765 cases with long-term follow-up. Am J Surg Pathol 2005; 29: 52-68.

19. Burton L Eisenberg, Ian Judson. Surgery and Imatinib in the Management of GIST: Emerging Approaches to Adjuvant and Neoadjuvant Therapy. Ann Surg Oncol 2004; 11: 465-75. 